\title{
Research Progress of Dopamine Receptors and Motor Control
}

\author{
Xiaoxin Wanga, Ke Lib ${ }^{\mathrm{b}}$, Jiali Cheng ${ }^{\mathrm{c}}$, Yanhang Zhang ${ }^{\mathrm{d}}$ and Lijuan $\mathrm{Hou}^{\mathrm{e}}$ \\ PE and Sports Science Department, Beijing Normal University, Beijing, 100875, China \\ a201521070030@mail.bnu.edu.cn, b18865382787@163.com, c405270197@qq.com, \\ d302590711@qq.com, ehoulj@bnu.edu.cn,
}

\begin{abstract}
Keywords: Dopamine; Basal Ganglia; DAR; PD; motor control;
Abstract. Dopamine receptors (DAR) involved in the regulation of many important functions in the brain as the targets of neurotransmitter dopamine(DA), especially in the aspects of motor control, learning and memory, cognition and drug addiction. But there are still a lot of controversies in the regulation of DAR in many neural circuits. In this review, we are focus on the regulation of DAR in the basal ganglia and summarizes the recent discoveries of DAR in motion control in recent years, these findings not only demonstrate the important role of DAR in the regulation of basal ganglia activity, but also serve as an important target for the treatment of basal ganglia diseases.
\end{abstract}

\section{Introduction}

The normal behavior of the human body is an external manifestation of the perfect coordination of the basal ganglia and cerebral cortex. It is only when there is a correct transmitter transmission, signal transmission between nucleuses, so that the human body shows no different from ordinary behavior. The basal ganglia are a group of interconnected nuclei, which play an important role in the regulation of movement and learning cognitive function [1-3]. As an important neurotransmitter, DA plays an important role in the regulation of the basal ganglia activity, movement and the spirit, and it is also a variety of important diseases (such as PD and $\mathrm{TH}$ ) therapeutic targets.

The signal transduction of DA is mediated by dopamine receptors. DAR can be divided into 5 subtypes and can be classified into two types: D1-like receptors (D1DR and D5DR) and D2-like receptors (D2DR, D3DR and D4DR). They belong to the family of G protein coupled receptors (GPCRs) with seven transmembrane structures. In recent years, with the continuous development and update of the technology of gene transfer, genetic and functional magnetic resonance, these technologies provides a good opportunity for the development of motor neurobiology. Therefore, the role of DAR in the basal ganglia in motor control has also been found. In this paper, we review these findings in order to have a deeper understanding of the role of DAR in motor control.

\section{DAR Participates in Basal Ganglia Loop Activity}

In the whole process of movement, the basal ganglia are an important central structure in the regulation of motor behavior. It is a general term for a group of interconnected subcortical nuclei, including the striatum, substantia nigra(SN), globus pallidus(GP) and subthalamic nucleus(STN). The basal ganglia mainly receive afferent projections from a wide area of the cerebral cortex, and the afferent connections from the cortex and substantia nigra were all terminated in the striatum (Fig. 1).

The striatum not only involved in the preparation and execution of voluntary movement, but also plays an important role in adjusting the direction, sequence, speed and amplitude of motion, as well as the plasticity of motion, such as habit formation and conditioned behavior, as well as cognitive memory and emotional motivation [4].

The main neuronal type of the striatum, making up approximately $95 \%$ of all neurons in the striatum, is the GABAergic medium-spiny projection neuron (MSN). It is the main target of the DA feedback system in the SNc and receives a lot of feedback information, can regulation of cortical and thalamic nucleus information through the basal ganglia circulation [5]. The rest of the neurons in the striatum are composed of a variety of cholinergic and GABA interneurons [6], can control the activities of MSNs locally. The imbalance of DA levels in the striatum can lead to motor dysfunction, 
abnormal gait and cognitive impairment, which has been confirmed in patients with Parkinson's disease and other neurodegenerative diseases [7].

The D1DR and D2DR-expressing MSNs are the projection neurons of the striatum and give rise to the so-called direct and indirect pathways, respectively depending on their projections to downstream nuclei of the basal ganglia [8,9]. Although the D2DR-expression MSNs usually has a stronger excitability and D1DR-expression MSNs has more dendritic branching, the basic parameters of the two types of projection neurons are highly overlapped, it is difficult to distinguish reliably according to their general morphology or basic electrophysiological properties [10,11]. In a study published in 2011 by Beaulieu et al., DA can activate the protein kinase A (PKA) though the D1DR and open the L- type calcium channel, and then participate in the regulation of motor control function in the striatum [12]. While Marcott et al. in 2014 proved that DA can also act on D2DR and activated potassium channel, and then caused D2DR inhibitory postsynaptic currents (IPSC), which makes the plasma membrane hyperpolarization and involvement in the regulation of motor function in the striatum [13]. In summary, DA has an excitatory effect on the direct pathway via D1DR and an inhibitory effect on the indirect pathway via D2DR, So that the striatum becomes the main nucleus of the motor function adjustment $[14,15]$.

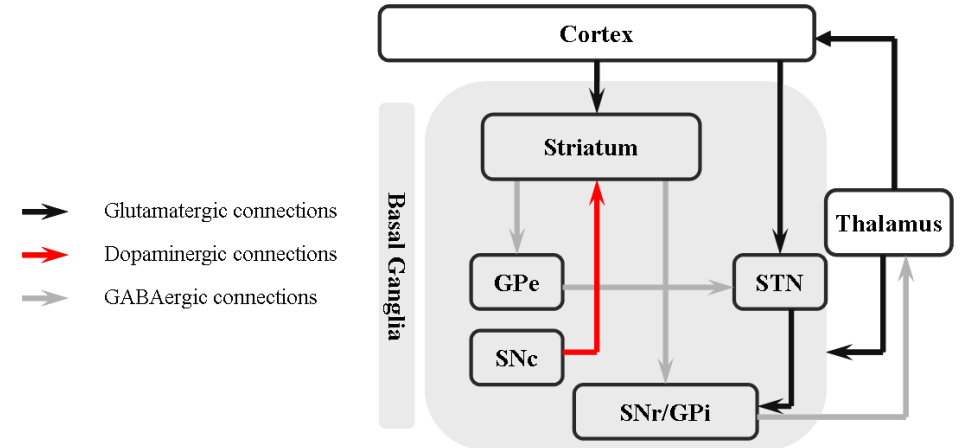

Fig. 1, Schematic diagram of basal ganglia circuit

D1DR is mainly distributed in the direct pathway: MSNs $\rightarrow \mathrm{GPi} / \mathrm{SNr}$.

D2DR is mainly distributed in the indirect pathway: MSNs $\rightarrow$ GPe $\rightarrow$ STN $\rightarrow$ GPi/SNr.

\section{DAR Participates in the Regulation of Somatic Movement}

The somatic motor system is a general term for the structure of motor control in the central nervous system, including the cerebral cortex, brainstem, spinal cord, basal ganglia, cerebellum, and peripheral motor nerve fibers.

D1DR receptors are located on the GABA and P nerve terminal which from the striatum into the $\mathrm{SNc}$ and the dendrites of DA neurons are distributed in the SNc. Dendritic release of DA regulates the activity of D1DR receptors in the SNc. The D2DR as presynaptic receptors is located in the SNc projecting to the striatum of the DA nerve fiber terminals, inhibit DA pulse flow and participate in the synthesis of DA release and reuptake process [16]; And expressed on the striatum MSNs nerve terminals and regulation of somatic motor function by descending pathway of the basal ganglia.

There was study showed that loss of cortical D1DR-expressing cells is sufficient to produce deficits in multiple motor and behavioral domains, independent of striatal mechanisms. Primary cortical changes in the D1DR compartment are therefore likely to model a number of core clinical features in disorders such as Huntington disease and schizophrenia [17]. In 2016, Bello et al study found that mice loss of D2DR during adulthood causes severe motor impairments, including hypolocomotion, deficits in motor coordination, impaired learning of new motor routines. Moreover, severe motor impairment, resting tremor and abnormal gait and posture, phenotypes reminiscent of Parkinson's disease, were evident when the mutation was induced in aged mice [18]. Similarly, there are studies showed that selective deletion of D2DR from indirect-pathway medium spiny neurons (iMSNs) is sufficient to impair locomotor activity, phenocopying DA depletion models of Parkinson's disease, despite this mouse model having intact DA transmission [19]. We already know 
that arsenic exposure can alterations in locomotor activity, behavioral tasks, and neurotransmitters systems in rodents. Leticia in the 2016 study showed that arsenic exposure produced hypoactivity at six months and other behaviors such as rearing and on-wall rearing and barbering showed both increases and decreases. No alterations on monoamines levels in striatum or frontal cortex were observed but a significant decrease in the expression of mRNA for D2DR was found in striatum of mice [20]. It is suggested that the abnormal motor behavior induced by arsenic exposure may be related to the level of D2DR.

In the midbrain, levels of D2DR mRNA were different between genetic groups with different endurance running capacities. And elevated levels of D2DR mRNA in the SNc and the VTA. But there was no significant difference in the levels of D1DR mRNA [21]. And in study of the model of exercise-induced fatigue in rats found that after exercise-induced fatigue, the DA nerve system in SNc mainly regulate electrical activity in striatum by D2DR [22], suggested that D2DR was involved in the central regulation of sports fatigue. The levels of DA in striatum of rats after one time exhaustive exercise were significantly increased (Fig. 2) [23], and D2DR levels were significantly increased, but D1DR did not change (Table 1) [24].
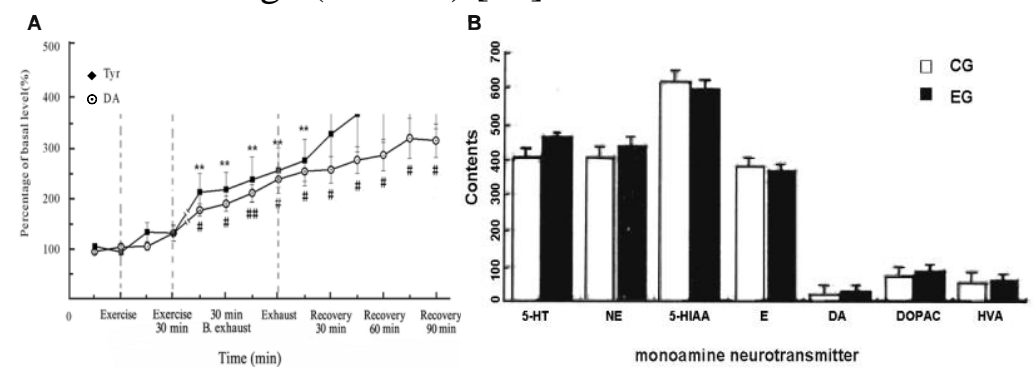

Fig. 2, Schematic diagram of monoamine neurotransmitters in model of exercise-fatigue rat A: The levels of DA and Tyr in striatum of rats after one time exhaustive exercise were significantly increased. B: Changes of monoamine neurotransmitters in striatum after repeated exhaustive exercise in rats.(CG means control group, EG means experiment group) (Previous work from our laboratory, references [23] and [24])

Table 1, Changes of D1DR and D2DR in striatum after repeated exhaustive exercise in rats

\begin{tabular}{ccl} 
Group & D1DR & \multicolumn{1}{c}{ D2DR } \\
\hline CG & 0.029549 & 0.01878272 \\
EG & 0.026201 & $0.04058527^{(1)}$
\end{tabular}

(1) means that compared with CG, P < 0.01. (Previous work from our laboratory, references [24])

\section{Conclusion}

The basal ganglia are an important central structure in the regulation of motor behavior, if it is lesioned or damaged will lead to severe dyskinesia. The high expression of D1DR and D2DR in the basal ganglia also played a key role in motor function. Used of progressive science and technology, scientists has found a lot of functional evidence related to DAR. But these technologies used in the field of sports research is relatively small. If we can take advantage of the current popular optical genetic technology, combined with functional magnetic resonance imaging and transgenic technology, can clarify the problems existing in the direct, indirect and even in super direct pathways involved in the movement disorders in the future, thus it will continue to serve sports and clinical medicine.

\section{Acknowledgements}

This study has been supported by the National Natural Science Foundation of China (31401018). National Training Programs of Innovation and Entrepreneurship for Undergraduates in 2016 
(201610027008). Associate professor Lijuan Hou is the corresponding author of this paper and her research interests include motor physiology and motor control.E-mail is houlj@bnu.edu.cn.

\section{References}

[1] A. M. Graybiel: Curr Opin Neurobiol Vol. 5 (1995), p. 733-741

[2] C. Hammond, H. Bergman and P. Brown: Trends Neurosci Vol. 30 (2007), p. 357-364

[3] T. Wichmann and M. R. Delong: Basal Ganglia Vol. 1 (2011), p. 65-77

[4] B. D. Devan, N.S. Hong and R. J. McDonald: Neurobiol Learn Mem Vol. 96 (2011), p. 95-120

[5] J. P. Bolam, M. T. C. Brown, J. Moss and P. J. Magill: Encyclopedia of Neuroscience (2009), p. 97-104

[6] N. Mallet, M. C. Le, S. Charpier and F Gonon: Journal of Neuroscience the Official Journal of the Society for Neuroscience Vol. 25 (2005) p. 3857-3869

[7] M. E. Rice, J. C. Patel and S. J. Cragg: Neuroscience Vol. 198 (2011), p. 112-137

[8] C. R. Gerfen, T. M. Engber, L. C. Mahan and Z. Susel et al.: Science Vol. 250 (1990), p. 1429-1432

[9] Y. Smith, M. D. Bevan, E. Shink and J. P. Bolam: Neuroscience Vol. 86 (1998), p. 353-387

[10] T. S. Gertler, C. S. Chan and D. J. Surmeier: Journal of Neuroscience the Official Journal of the Society for Neuroscience Vol. 28 (2008), p. 10814-10824

[11] A. C. Kreitzer: Annual Review of Neuroscience Vol. 32 (2009), p. 127-147

[12] J. M. Beaulieu and R. R. Gainetdinov:Pharmaclolgical Reviews Vol. 63 (2011), p. 182-217

[13] P. P. Maarcott, A. A. Mamaligas and C. P. Ford: Neuron Vol. 84 (2014), p. 164-176

[14] B. J. Paul and T. J. Ellender: Neuropharmacology Vol. 106 (2015), p. 74-84

[15] C. R. Gerfen and D. J. Surmeier: Annual Review of Neuroscience Vol. 34 (2011), p. 441

[16] Y. Yao and H. W. Ma: Foreign Med Sci Pediatr Vol. 32 (2005), p. 137-140

[17] L. Jiang, C. O'Leary, H. A. Kim and C. L. Parish: Neurobiology of Disease Vol. 76 (2015), p. 137

[18] E. P. Bello, R. Casascordero, G. L. Galiñanes and E. Casey et al.: Molecular Psychiatry (2016)

[19] J. C. Lemos, D. M. Friend, A. R. Kaplan and J. H. Shin et al.: Neuron Vol. 90 (2016), p. 824-838

[20] M. Á. C. Leticia, J. H. Limón-Pacheco, G. Magda and V. M. Rodríguez: Journal of Toxicology Vol. 2016 (2016), p. 1-10

[21] T. E. Foley, B. N. Greenwood, H. E. Day and L. G. Koch et al.: Behavioural Brain Research Vol. 174 (2006), p. 132-142

[22] X. L. Liu, D. Wu, D. C. Qiao and L. J. Hou: Journal of Xian Physical Education University Vol. 29 (2012), p. 78-83

[23] D. S. Yang, X. L. Liu and D. C. Qiao: Chinese Journal of Applied Physiology Vol. 4 (2011), p. 432-436

[24] L. J. Hou, X. L. Liu and D. C. Qiao: Chinese Journal of Rehabilitation Medicine Vol. 25 (2010), p. $639-642$ 